\title{
A NON-AUTONOMOUS STRONGLY DAMPED WAVE EQUATION: EXISTENCE AND CONTINUITY OF THE PULLBACK ATTRACTOR
}

TOMÁS CARABALLO ${ }^{1}$, ALEXANDRE N. CARVALHO $^{2}$, JOSÉ A. LANGA ${ }^{1}$, AND FELIPE RIVERO ${ }^{1}$

\begin{abstract}
In this paper we consider the strongly damped wave equation with time dependent terms

$$
u_{t t}-\Delta u-\gamma(t) \Delta u_{t}+\beta_{\varepsilon}(t) u_{t}=f(u)
$$

in a bounded domain $\Omega \subset \mathbb{R}^{n}$, under some restrictions on $\beta_{\varepsilon}(t), \gamma(t)$ and growth restrictions on the non-linear term $f$. The function $\beta_{\varepsilon}(t)$ depends on a parameter $\varepsilon, \beta_{\varepsilon}(t) \stackrel{\varepsilon \rightarrow 0}{\longrightarrow} 0$. We will prove, under suitable assumptions, local and global well posedness (using the uniform sectorial operators theory), the existence and regularity of pullback attractors $\left\{\mathscr{A}_{\mathcal{E}}(t)\right.$ : $t \in \mathbb{R}\}$, uniform bounds for these pullback attractors, characterization of these pullback attractors and their upper and lower semicontinuity at $\epsilon=0$.
\end{abstract}

\section{Introduction}

Let us consider the equation

$$
\left\{\begin{array}{l}
u_{t t}-\Delta u-\gamma(t) \Delta u_{t}+\beta_{\varepsilon}(t) u_{t}=f(u) \text { in } \Omega, \\
u=0 \text { on } \partial \Omega
\end{array}\right.
$$

in a sufficiently smooth bounded domain $\Omega \subset \mathbb{R}^{n}, \gamma, \beta_{\varepsilon}: \mathbb{R} \rightarrow(0, \infty)$ verifies $0<\gamma_{0} \leqslant \gamma(t) \leqslant$ $\gamma_{1}<\infty, 0<\beta_{0 \varepsilon} \leqslant \beta(t) \leqslant \beta_{1 \varepsilon}<\infty$, and $\gamma(t)$ and $\beta_{\varepsilon}(t)$ are continuously differentiable in $\mathbb{R}$ and with bounded derivative uniformly in $\epsilon$. We also suppose that

$$
\begin{aligned}
& \beta_{i \varepsilon} \stackrel{\varepsilon \rightarrow 0}{\longrightarrow} 0, \text { for } i=0,1 \\
& \frac{\beta_{1 \varepsilon}}{\beta_{0 \varepsilon}} \stackrel{\varepsilon \rightarrow 0}{\longrightarrow} 1 .
\end{aligned}
$$

For the nonlinearity $f: \mathbb{R} \rightarrow \mathbb{R}$ we assume that it verifies the following dissipativeness and growth conditions

$$
\begin{aligned}
& f \in C^{2}(\mathbb{R}, \mathbb{R}), \quad \limsup _{|s| \rightarrow \infty} \frac{f(s)}{s} \leqslant 0, \\
& \left|f^{\prime \prime}(s)\right| \leqslant c\left(1+|u|^{\rho-2}\right)
\end{aligned}
$$

where $1<\rho<\frac{n+2}{n-2}$.

\footnotetext{
${ }^{1}$ Partially supported by Ministerio de Ciencia e Innovación grant \# MTM2008-0088 and Junta de Andalucía grants \# P07-FQM-02468, \# FQM314 and HF2008-0039, Spain.

${ }^{2}$ Partially supported by CNPq 305447/2005-0 and 451761/2008-1, CAPES/DGU 267/2008 and FAPESP 2008/53094-4, Brazil; Ministerio de Ciencia e Innovación grant \# MTM2008-0088, and Junta de Andalucía grant \# P07-FQM-02468,
} 
The aim of this paper is to obtain the existence, regularity, continuity, characterization and continuity of characterization of pullback attractors for the problem (1) in $H_{0}^{1}(\Omega) \times L^{2}(\Omega)$. In particular, for the characterization of the pullback attractors we obtain that (when all the equilibria are hyperbolic) all solutions in the pullback attractors are backwards and forwards asymptotic to equilibria. We also obtain that the evolution process associated to (11) in $H_{0}^{1}(\Omega) \times L^{2}(\Omega)$ is a gradient-like evolution process (homoclinic structures do not exist).

There are some relevant physical applications for this kind of equation. In space dimensions $n=1$ and $n=2$, if $\gamma(t) \equiv 1$ and $\beta_{\varepsilon}(t) \equiv 0$, equation (11) models the longitudinal vibrations of a homogeneous bar subjected to viscous effects. The term $-\Delta u_{t}$ indicates that the stress is proportional to the strain rate as in a linearized Kelvin-Voigt material. In dimension three, the model describes the variation from the configuration at rest of a homogeneous and isotropic linear viscoelastic solid with short memory, called rate type (see [17]), in the presence of an external displacement-dependent force. The term $\beta u_{t}$ with $\beta>0$ indicates that the bar is subjected to dynamical friction as well. We also have a perturbed sine-Gordon equation of the form $u_{t t}-\alpha \Delta u_{t}-\Delta u+\sin u+\beta u_{t}=f(u)$, describing the evolution of the current $u$ in a Josephson junction (see [16]).

This equation is a generalization of an autonomous model which has been investigated in many articles by several authors (see, for example, [6, 7, 3, 13, 14, 21, 24, 25), with particular emphasis on its asymptotic behaviour. Assuming (critical) growth restrictions on the non-linear term $f$, the global existence of solutions in the autonomous case has been established in [6], based on the theory of $\varepsilon$-regular solutions.

To better explain the results in the paper we introduce the terminology of evolution processes. Our framework will be that of evolution processes for non-autonomous dynamical systems (Cheban [12], Chepyzhov-Vishik [15], Kloeden [22], Sell [26]). An evolution process in a Banach space $\mathcal{Z}$ is a family of maps $\{S(t, s): t \geqslant s\}$ from $\mathcal{Z}$ into itself with the following properties:

1) $S(t, t)=I$, for all $t \in \mathbb{R}$,

2) $S(t, s)=S(t, \tau) S(\tau, s)$, for all $t \geqslant \tau \geqslant s$,

3) $\left\{(t, s) \in \mathbb{R}^{2}: t \geqslant s\right\} \times \mathcal{Z} \ni(t, s, x) \mapsto S(t, s) x \in \mathcal{Z}$ is continuous.

A family of sets $\{K(t): t \in \mathbb{R}\}$ pullback attracts bounded subsets of $\mathcal{Z}$ under $\{S(t, s): t \geqslant$ $s\}$ if $K(t)$ pullback attracts all bounded subsets at $t$ under $\{S(t, s): t \geqslant s\}$, for each $t \in \mathbb{R}$, i.e., for each bounded subset $C \subset \mathcal{Z}$,

$$
\lim _{s \rightarrow-\infty} \operatorname{dist}(S(t, s) C, K(t))=0,
$$

where $\operatorname{dist}(\cdot, \cdot)$ denotes the Hausdorff semi-distance,

$$
\operatorname{dist}(A, B)=\sup _{a \in A} \inf _{b \in B}|a-b| .
$$

An evolution process $\{S(t, s): t \geqslant s\}$ is said to be pullback strongly bounded dissipative if, for each $t \in \mathbb{R}$, there exists a bounded subset $B(t)$ of $\mathcal{Z}$ which pullback attracts bounded subsets of $\mathcal{Z}$ at time $\tau$, for each $\tau \leqslant t$. In other words, given a bounded subset $B$ of $\mathcal{Z}$ and $\tau \leqslant t$, there exists $s_{0}(\tau, D)$ such that $S(\tau, s) B \subset B(t)$, for all $s \leqslant s_{0}(\tau, D)$. 
A family $\{\mathscr{A}(t): t \in \mathbb{R}\}$ of compact subsets of $\mathcal{Z}$ is said to be the pullback attractor for the evolution process $\{S(t, s): t \geqslant s\}$ if it is invariant $(S(t, s) \mathscr{A}(s)=\mathscr{A}(t)$ for all $s \leqslant t)$, pullback attracts bounded subsets of $\mathcal{Z}$, and is minimal, that is, if there is another family of closed sets $\{C(t): t \in \mathbb{R}\}$ which pullback attracts bounded subsets of $\mathcal{Z}$, then $A(t) \subseteq C(t)$, for all $t \in \mathbb{R}$.

In Section 2 we will use the theory of uniform sectorial operators, as in [11, 27, to prove the local well-posedness and regularity for (11) in $H_{0}^{1}(\Omega) \times L^{2}(\Omega)$. The existence of the attractor is proved in Section 3 using results based on asymptotic compact processes that appear in [4] and using an energy functional to obtain some useful estimates. In Section 4 , following the ideas in [5] (see also [25]), we show that the pullback attractor is a bounded set in $H^{2}(\Omega) \times H^{2}(\Omega)$. In Section 5 it is shown how the pullback attractor of the limit problem is a gradient-like attractor, that is, all the bounded global solutions converge backwards and forwards to some fixed point of the system, and then the attractor can be characterized as the union of unstable manifolds of equilibria. Finally, in Section 6 we will prove the continuity of the attractors when $\varepsilon \rightarrow 0$. To this end, we first show the upper-semicontinuity using standard techniques based on the continuity of the processes, and then follow [9] (see also [8, 10]) to obtain the lower semicontinuity. One can find some particular cases containing time-dependent damped terms, for example, in [20, 23] (see also the references therein).

\section{EXISTENCE OF SOLUTION}

To obtain the local well posedness of the Cauchy problem for (1) we will use the results in [11] (see also, [27]) which we introduce next

Definition 2.1. Let $\mathcal{Z}$ be a Banach space and $B(t): D \subset \mathcal{Z} \rightarrow \mathcal{Z}$ (D fixed) a closed, densely defined, time-dependent operator.

a) The operator family $B(t)$ is called uniformly sectorial if there is a constant $C>0$ (independent of $t \in \mathbb{R}$ ) such that

$$
\left\|(\lambda I+B(t))^{-1}\right\|_{\mathcal{L}(\mathcal{Z})} \leqslant \frac{C}{|\lambda|+1} ; \forall \lambda \in \mathbb{C} \text { with } \operatorname{Re} \lambda \geqslant 0 .
$$

b) $B(t)$ is called uniformly Hölder continuous if there exist constants $C>0$ and $\varepsilon>0$ such that, for any $t, \tau, s \in \mathbb{R}$,

$$
\left\|[B(t)-B(\tau)] B^{-1}(s)\right\|_{\mathcal{L}(\mathcal{Z})} \leqslant C(t-\tau)^{\varepsilon} .
$$

Consider the Cauchy problem

$$
\begin{aligned}
& z_{t}=B(t) z+F(z), t>t_{0}, \\
& z\left(t_{0}\right)=z_{0} .
\end{aligned}
$$

Definition 2.2. For a continuous function $F: \mathbb{R} \times \mathcal{Z}^{\alpha} \rightarrow \mathcal{Z}, \alpha \in[0,1), z\left(\cdot, t_{0}, z_{0}\right)$ : $\left[t_{0}, t_{0}+\tau\right) \rightarrow \mathcal{Z}^{\alpha}$ is a solution for (5) if it is continuous, continuously differentiable in $(0, \tau)$, $z\left(t, t_{0}, z_{0}\right) \in D(B(t))$ for $t \in\left(t_{0}, t_{0}+\tau\right)$ and (5) is satisfied for all $t \in(0, \tau)$.

We can now state the following result (see [11] for a more general version that includes the critical growth case). 
Theorem 2.3. If the operator family $B(t)$ is uniformly sectorial and uniformly Hölder continuos, and $F: \mathcal{Z}^{\alpha} \rightarrow \mathcal{Z}$ is Lispschitz continuous in bounded subsets of $\mathcal{Z}^{\alpha}$ then, given $r>0$ there exists $\tau>0$ and for each $z_{0} \in \mathcal{Z}^{\alpha}$ with $\left\|z_{0}\right\|_{\mathcal{Z}^{\alpha}} \leqslant r$ a function $z\left(\cdot, t_{0}, z_{0}\right) \in$ $C\left(\left[t_{0}, t_{0}+\tau\right], \mathcal{Z}^{\alpha}\right) \cap C^{1}\left(\left(t_{0}, t_{0}+\tau\right], \mathcal{Z}^{\alpha}\right)$ with the properties that

$$
\left\{z_{0} \in \mathcal{Z}^{\alpha}:\left\|z_{0}\right\|_{\mathcal{Z}^{\alpha}} \leqslant r\right\} \ni z_{0} \mapsto z\left(\cdot, t_{0}, z_{0}\right) \in C\left(\left[t_{0}, t_{0}+\tau\right], \mathcal{Z}^{\alpha}\right)
$$

is continuous, $z\left(\cdot, t_{0}, z_{0}\right)$ is the unique solution of (5).

It is clear from this result (since the time of existence can be chosen uniform in bounded subsets of $\mathcal{Z}^{\alpha}$ ) that solutions that do not blow up in the $\mathcal{Z}^{\alpha}$ must exist for all $t \geqslant t_{0}$.

Next we establish the functional analytic framework needed to apply the above results to the problem (11). Let $\Omega \subset \mathbb{R}^{n}$ be a bounded smooth domain, $X=L^{2}(\Omega)$ and $A$ : $D(A) \subset X \rightarrow X$ be the positive self-adjoint operator defined by $D(A)=H^{2}(\Omega) \cap H_{0}^{1}(\Omega)$ and $A u=-\Delta u$ for all $u \in D(A)$. Let $A^{\alpha}$, with $\alpha \in \mathbb{R}$, be the fractional power operators associated to $A$ and, for $\alpha \geqslant 0$, let $X^{\alpha}=D\left(A^{\alpha}\right)$, with the norm $\|\cdot\|_{\alpha}=\left\|A^{\alpha} \cdot\right\|_{X}$, be the fractional power spaces associated to $A$ for $\alpha \geqslant 0$. Define $X^{-\alpha}, \alpha>0$, as the completion of $X$ with respect to the norm $\left\|A^{-\alpha} \cdot\right\|_{X}$. With this notation, we will keep denoting by $A$ the closed extension of $A$ to $X^{-\alpha}, \alpha \geqslant 0$. Finally, denote by $Y_{(-1)}=X^{\frac{1}{2}} \times X^{-\frac{1}{2}}$

With this, we can view (1) as the system

$$
\left[\begin{array}{l}
u \\
v
\end{array}\right]_{t}+\mathbf{A}(t)\left[\begin{array}{l}
u \\
v
\end{array}\right]=F\left(\left[\begin{array}{l}
u \\
v
\end{array}\right]\right)
$$

where $D(\mathbf{A}(\mathbf{t}))=X^{\frac{1}{2}} \times X^{\frac{1}{2}}$

$$
\mathbf{A}(\mathbf{t})\left[\begin{array}{l}
\phi \\
\varphi
\end{array}\right]=\left[\begin{array}{cc}
0 & -I \\
A & \gamma(t) A+\beta_{\varepsilon}(t)
\end{array}\right]\left[\begin{array}{l}
\phi \\
\varphi
\end{array}\right]:=\left[\begin{array}{c}
-\varphi \\
A(\phi+\gamma(t) \varphi)+\beta_{\epsilon}(t) \varphi
\end{array}\right],
$$

and

$$
F\left(\left[\begin{array}{l}
u \\
z
\end{array}\right]\right)=\left[\begin{array}{c}
0 \\
\mathbf{f}(u)
\end{array}\right]
$$

where, for $\phi: \Omega \rightarrow \mathbb{R}, \mathbf{f}(\phi)$ is defined by $\mathbf{f}(\phi)(x)=f(\phi(x)), x \in \Omega$.

Note that $D(\mathbf{A}(\mathbf{t}))$ (as a vector space) does not depend on $t$. Denote by $Y_{(-1)}^{1}$ the space $X^{\frac{1}{2}} \times X^{\frac{1}{2}}$ with the norm $\|\mathbf{A}(t) \cdot\|_{Y_{(-1)}}$, where

$$
\mathbf{A}(t)=\left[\begin{array}{cc}
0 & -I \\
A & \gamma(t) A+\beta_{\epsilon}(t) I
\end{array}\right] .
$$

It is easy to see that $Y_{(-1)}^{1}$ is isomorphic to $X^{\frac{1}{2}} \times X^{\frac{1}{2}}$ with its usual norm, uniformly for $t \in \mathbb{R}$. Also note that,

$$
\mathbf{A}(t)^{-1}=\left[\begin{array}{cc}
\gamma(t) I+\beta_{\epsilon}(t) A^{-1} & A^{-1} \\
-I & 0
\end{array}\right] .
$$

Denote by $Y_{(-1)}^{\alpha}$ the domain of $\mathbf{A}\left(t_{0}\right)^{\alpha}$ for some $t_{0} \in \mathbb{R}$ and $\alpha \geqslant 0$ with the graph norm; we obtain the fractional power scale spaces associated to $\mathbf{A}_{-1}^{\alpha}\left(t_{0}\right)$ (see[18]). As in [6], we can prove that the following embeddings hold (the operator $\mathbf{A}(t)$ is acretive and, as a consequence 
of that, it will have bounded imaginary powers, and hence the fractional power spaces will coincide with the interpolation spaces):

$$
\left\{\begin{array}{l}
Y_{(-1)}^{\alpha} \subset H^{1}(\Omega) \times H^{2 \alpha-1}(\Omega) \subset L^{q_{1}}(\Omega) \times L^{q_{2}}(\Omega) \\
\text { for } 1 \leqslant q_{1} \leqslant \frac{2 n}{n-2}, \quad 1 \leqslant q_{2} \leqslant \frac{2 n}{n-2(2 \alpha-1)}, \quad \alpha \in[0,1], \quad n \geqslant 3 .
\end{array}\right.
$$

Using this we establish the following local well posedness result for (6).

Theorem 2.4. The operator family $\mathbf{A}(t)$ is uniformly sectorial and uniformly Hölder continuos, and $F: \mathbb{R} \times Y_{(-1)}^{\frac{1}{2}} \rightarrow Y_{(-1)}$ defined by $F\left(\left[\begin{array}{l}u \\ v\end{array}\right]\right)=\left[\begin{array}{c}0 \\ \mathbf{f}(u)\end{array}\right]$ is Lispschitz continuous in bounded subsets of $Y_{(-1)}^{\frac{1}{2}}$. Then, given $r>0$ there exists $\tau>0$ and for each $U_{0} \in Y_{(-1)}^{\frac{1}{2}}$ with $\left\|U_{0}\right\|_{Y_{(-1)}^{\frac{1}{2}}} \leqslant r$ a function $U\left(\cdot, t_{0}, U_{0}\right) \in C\left(\left[t_{0}, t_{0}+\tau\right], Y_{(-1)}^{\frac{1}{2}}\right) \cap C^{1}\left(\left(t_{0}, t_{0}+\tau\right], Y_{(-1)}^{\frac{1}{2}}\right)$ with the properties that

$$
\left\{U_{0} \in Y_{(-1)}^{\frac{1}{2}}:\left\|U_{0}\right\|_{Y_{(-1)}^{\frac{1}{2}}} \leqslant r\right\} \ni U_{0} \mapsto U\left(\cdot, t_{0}, U_{0}\right) \in C\left(\left[t_{0}, t_{0}+\tau\right], Y_{(-1)}^{\frac{1}{2}}\right)
$$

is continuous, $U\left(\cdot, t_{0}, U_{0}\right)$ is the unique solution of (6) (in the sense of Definition (2.2) satisfying $U\left(t_{0}, t_{0}, U_{0}\right)=U_{0}$. If $U_{0} \in X^{1} \times X^{1}$, then $U(\cdot)$ is twice continuously differentiable in $(0, \tau)$ with values in $X^{\frac{1}{2}} \times X$.

The proof of this theorem consists on the verification of the assumptions of Theorem 2.3 and the proof of the the time regularity in the last statement of it. First we prove the uniform sectoriality and the uniform Hölder continuity of $\mathbf{A}(\mathbf{t})$. First note that

$$
(\lambda+\mathbf{A}(t))^{-1}=\left(\begin{array}{cc}
\left(\left(\lambda+\beta_{\epsilon}(t)\right) I+\gamma(t) A\right) R(\lambda) & R(\lambda) \\
-A R(\lambda) & \lambda R(\lambda)
\end{array}\right)
$$

where $R(\lambda)=\left(\lambda\left(\lambda+\beta_{\epsilon}(t)\right) I+(1+\lambda \gamma(t)) A\right)^{-1}$. With this, it is easy to check that

$$
\left\|(\lambda+\mathbf{A}(t))^{-1}\right\|_{\mathcal{L}\left(Y_{(-1)}\right)} \leqslant \frac{C}{|\lambda|+1} .
$$

Now,

$$
[\mathbf{A}(t)-\mathbf{A}(s)] \mathbf{A}\left(t_{0}\right)^{-1}=-(\gamma(t)-\gamma(s))\left(\begin{array}{cc}
0 & 0 \\
A & 0
\end{array}\right)-\left(\beta_{\epsilon}(t)-\beta_{\epsilon}(s)\right)\left(\begin{array}{ll}
0 & 0 \\
I & 0
\end{array}\right) .
$$

From this and from the Hölder continuity of $\gamma$ and $\beta_{\epsilon}$ uniformly in $\mathbb{R}$ and in $\epsilon$, it is clear that

$$
\left\|[\mathbf{A}(t)-\mathbf{A}(s)] \mathbf{A}\left(t_{0}\right)^{-1}\right\|_{\mathcal{L}\left(Y_{(-1)}\right)} \leqslant M|t-s|^{\epsilon}
$$

which proves the uniform Hölder continuity condition $b)$.

Lemma 2.5. If $f: \mathbb{R} \rightarrow \mathbb{R}$ satisfies (4) then,

$$
\begin{aligned}
\| \mathbf{f}\left(w_{1}\right) & -\mathbf{f}\left(w_{2}\right) \|_{L^{\frac{2 n}{n+2}}} \\
& \leqslant c\left\|w_{1}-w_{2}\right\|_{H^{1}}\left(1+\left\|w_{1}\right\|_{H^{1}}^{\rho-1}+\left\|w_{2}\right\|_{H^{1}}^{\rho-1}\right), w_{1}, w_{2} \in H^{1}(\Omega) .
\end{aligned}
$$


Proof: From (4), the Hölder inequality, and the Sobolev embeddings we obtain:

$$
\begin{aligned}
& \left\|\mathbf{f}\left(w_{1}\right)-\mathbf{f}\left(w_{2}\right)\right\|_{L^{\frac{2 n}{n+2}}(\Omega)} \\
& \leqslant c\left[\int_{\Omega}\left[\left|w_{1}-w_{2}\right|\left(1+\left|w_{1}\right|^{\rho-1}+\left|w_{2}\right|^{\rho-1}\right)\right]^{\frac{2 n}{n+2}}\right]^{\frac{n+2}{2 n}} \\
& \leqslant c\left\|w_{1}-w_{2}\right\|_{L^{\frac{2 n}{n-2}(\Omega)}}\left\|1+\left|w_{1}\right|^{\rho-1}+\left|w_{2}\right|^{\rho-1}\right\|_{L^{\frac{n}{2}}(\Omega)} \\
& \leqslant \tilde{c}\left\|w_{1}-w_{2}\right\|_{L^{\frac{2 n}{n-2}(\Omega)}}\left(1+\left\|w_{1}\right\|_{L^{\frac{n(\rho-1)}{2}}(\Omega)}^{\rho-1}+\left\|w_{2}\right\|_{L^{\frac{n(\rho-1)}{2}}(\Omega)}^{\rho-1}\right) .
\end{aligned}
$$

Since

$$
\frac{2 n(\rho-1)}{4} \leqslant \frac{2 n}{n-2}
$$

we have that

$$
L^{\frac{n(\rho-1)}{2}}(\Omega) \supset H^{1}(\Omega) .
$$

Which completes the proof.

This proves the following result.

Lemma 2.6. If $f: \mathbb{R} \rightarrow \mathbb{R}$ satisfies the above conditions then,

$$
\begin{aligned}
& \left\|F\left(\begin{array}{l}
\phi_{1} \\
\psi_{1}
\end{array}\right)-F\left(\begin{array}{l}
\phi_{2} \\
\psi_{2}
\end{array}\right)\right\|_{Y_{(-1)}} \leqslant c\left\|\left(\begin{array}{l}
\phi_{1} \\
\psi_{1}
\end{array}\right)-\left(\begin{array}{l}
\phi_{2} \\
\psi_{2}
\end{array}\right)\right\|_{Y_{(-1)}^{\frac{1}{2}}} \\
& \leqslant c\left(1+\left\|\left(\begin{array}{c}
\phi_{1} \\
\psi_{1}
\end{array}\right)\right\|_{Y_{(-1)}^{\frac{1}{2}}}^{\rho-1}+\left\|\left(\begin{array}{c}
\phi_{2} \\
\psi_{2}
\end{array}\right)\right\|_{Y_{(-1)}-1}^{\rho-1}\right),\left(\begin{array}{l}
\phi_{1} \\
\psi_{1}
\end{array}\right),\left(\begin{array}{c}
\phi_{2} \\
\psi_{2}
\end{array}\right) \in Y_{(-1)}^{\frac{1}{2}} .
\end{aligned}
$$

From all this and from Theorem 2.3 follows all assertions of Theorem 2.4 except the last one. To see that if $U_{0} \in X^{1} \times X^{1}, U\left(\cdot, U_{0}\right) \in C^{2}\left((0, \tau), X^{\frac{1}{2}} \times X\right)$ we apply again Theorem 2.3 to the following Cauchy problem

$$
\begin{gathered}
\frac{d}{d t}\left[\begin{array}{l}
u \\
v \\
w
\end{array}\right]+\mathcal{A}(t)\left[\begin{array}{l}
u \\
v \\
w
\end{array}\right]=\bar{F}\left[\begin{array}{l}
u \\
v \\
w
\end{array}\right], t>t_{0} \\
{\left[\begin{array}{c}
u\left(t_{0}\right) \\
v\left(t_{0}\right) \\
w\left(t_{0}\right)
\end{array}\right]=\left[\begin{array}{c}
u_{0} \\
v_{0} \\
-A u_{0}-\gamma^{\prime}\left(t_{0}\right) A v_{0}-\beta_{\epsilon}\left(t_{0}\right) v_{0}+\mathbf{f}\left(u_{0}\right)
\end{array}\right]}
\end{gathered}
$$

with $\mathcal{Z}=X^{1} \times X^{1} \times X, \mathcal{A}(t): D(\mathcal{A}(t)) \subset \mathcal{Z} \rightarrow \mathcal{Z}, D(\mathcal{A}(t))=X^{1} \times X^{1} \times X^{1}$

$$
\mathcal{A}(t)=\left[\begin{array}{ccc}
0 & -I & 0 \\
0 & 0 & -I \\
0 & \left(1+\gamma^{\prime}(t)\right) A+\beta_{\epsilon}^{\prime}(t) I & \gamma(t) A+\beta_{\epsilon}(t) I
\end{array}\right] \quad \text { and } \quad \bar{F}\left[\begin{array}{c}
u \\
v \\
w
\end{array}\right]=\left[\begin{array}{c}
0 \\
0 \\
\overline{\mathbf{f}}(u, v)
\end{array}\right]
$$


with $\mathbf{f}(u, v)(x):=f^{\prime}(u(x)) v(x)$. Firstly, we need to rewrite the problem since $\mathcal{A}(t)$ is singular and is not invertible. Therefore, we consider

$$
\frac{d}{d t}\left[\begin{array}{l}
u \\
v \\
w
\end{array}\right]+\mathcal{C}(t)\left[\begin{array}{c}
u \\
v \\
w
\end{array}\right]=\bar{H}\left[\begin{array}{c}
u \\
v \\
w
\end{array}\right]
$$

with

$$
\mathcal{C}(t)=\left[\begin{array}{ccc}
-I & 0 & 0 \\
0 & 0 & -I \\
0 & \left(N+\gamma^{\prime}(t)\right) A+\beta_{\epsilon}^{\prime}(t) I & \gamma(t) A+\beta_{\epsilon}(t) I
\end{array}\right] \quad \text { and } \quad \bar{H}\left[\begin{array}{c}
u \\
v \\
w
\end{array}\right]=\left[\begin{array}{c}
u-v \\
0 \\
\overline{\mathbf{h}}(u, v)
\end{array}\right]
$$

with $\mathbf{h}(u, v)(x):=(1+N) A v+f^{\prime}(u(x)) v(x)$ and $N$ big enough. Then, there is a unique solutions which is a continuously differentiable function with values in $\mathcal{Z}=X^{1} \times X^{1} \times X$ since $\overline{\mathbf{h}}: \mathcal{Z} \rightarrow \mathcal{Z}$ is Lypschitz in bounded subsets of $\mathcal{Z}$ and $\mathcal{C}(t)$ easily satisfies all properties of Theorem 2.3. Also, using techniques similar to those used to study $\mathbf{f}$ in Lemma 2.6, we obtain properties of the nonlinearity $\overline{\mathbf{h}}: X^{1} \times X^{1} \times X \rightarrow X$ that ensure that $\bar{H}$ also satisfies the hypothesis of Theorem 2.3 and proves the last statement of Theorem 2.4

Remark 2.7. Note that, if $u \in X^{\frac{1}{2}}$ and following Lemma 2.5, f $(u) \in L^{\frac{2 n}{n+2-2 \epsilon}} \hookrightarrow X^{-\frac{1}{2}+\epsilon}$ for some $\epsilon>0$ such that

$$
0<\epsilon=\frac{n(2-\rho)}{2}+\rho .
$$

Hence, we may obtain that the solutions given in Theorem 2.4 with initial data in $X^{\frac{1}{2}+\epsilon} \times X^{\epsilon}$ are in fact in $X^{\frac{1}{2}+\epsilon} \times X^{\frac{1}{2}+\epsilon}$ for $t \in\left(t_{0}, t_{0}+\tau\right)$. We can repeat this reasoning, noting that if $u \in X^{\frac{1}{2}+\epsilon}$, then $\mathbf{f}(u) \in X^{-\frac{1}{2}+\rho \epsilon}$ and, after a finite number of steps, we obtain that solutions with initial data in $X^{1} \times X^{\frac{1}{2}}$ are in $X^{1} \times X^{1}$.

\section{EXISTENCE OF PULlbaCK ATTRACTORS}

First we need to recall some definitions and properties which will be needed in this section. An evolution process $\{S(t, s): t \geqslant s\}$ in a Banach space $Z$ is said pullback asymptotically compact if, for each $t \in \mathbb{R}$, each sequence $\left\{s_{k}\right\}_{k \in \mathbb{N}}$ in $(-\infty, t]$ such that $s_{k} \stackrel{k \rightarrow \infty}{\longrightarrow}-\infty$, and each bounded sequence $\left\{z_{k}\right\}_{k \in \mathbb{N}}$ in $Z$ with $\left\{S\left(t, s_{k}\right) z_{k}: k \in \mathbb{N}\right\}$ bounded, the sequence $\left\{S\left(t, s_{k}\right) z_{k}\right\}_{k \in \mathbb{N}}$ possesses a convergent subsequence. It is said that $\{S(t, s): t \geqslant s\}$ is pullback strongly bounded if, for each $t \in \mathbb{R}$ and each bounded subset $B$ of $Z, \bigcup_{s \leqslant t} \bigcup_{\tau \leqslant s} S(s, \tau) B$ is bounded. Now we state the followings results from [5], which will lead to the existence of the pullback attractor for (6) $)$.

Theorem 3.1. If an evolution process $\{S(t, s): t \geqslant s\}$ is pullback strongly bounded dissipative and pullback asymptotically compact, then $\{S(t, s): t \geqslant s\}$ possesses a pullback attractor $\{\mathscr{A}(t): t \in \mathbb{R}\}$ with the property that $\bigcup_{s \leqslant t} \mathscr{A}(s)$ is bounded for each $t \in \mathbb{R}$.

Theorem 3.2. Let $\{S(t, s): t \geqslant s\}$ be a pullback strongly bounded process in a Banach space $Z$ such that $S(t, s)=T(t, s)+U(t, s)$, where $U(t, s)$ is compact and there exists a non-increasing function $k: \mathbb{R}^{+} \times \mathbb{R}^{+} \longrightarrow \mathbb{R}$ with $k(\sigma, r) \rightarrow 0$ when $\sigma \rightarrow \infty$, and for all $s \leqslant t$ 
and $z \in Z$ with $\|z\| \leqslant r,\|T(t, s) z\| \leqslant k(t-s, r)$. Then the process $\{S(t, s): t \geqslant s\}$ is pullback asymptotically compact.

From now on, we denote by $Y^{0}:=H_{0}^{1}(\Omega) \times L^{2}(\Omega)$, which will be the phase space or our process (notice that with the notation used in Section [2, $Y^{0}=X^{1 / 2} \times X$ which is isomorphic to $\left.Y_{(-1)}^{\frac{1}{2}}\right)$.

For $b \in \mathbb{R}^{+}$, let us define $L_{b}(\cdot, \cdot): Y^{0} \longrightarrow \mathbb{R}$ as

$$
L_{b}(\phi, \varphi)=\frac{1}{2}\|\phi\|_{H_{0}^{1}}^{2}+\frac{1}{2}\|\varphi\|_{L^{2}}^{2}+b(\phi, \varphi)_{L^{2}}-\int_{\Omega} G(\phi) d x,
$$

with $G(s)=\int_{0}^{s} f(\theta) d \theta$.

If $\frac{b}{2} \lambda_{1}^{-1} \leqslant \frac{1}{4}$ and $b \leqslant \frac{1}{2}$, we have that

$$
\frac{1}{4}\left[\|\varphi\|_{H_{0}^{1}}^{2}+\|\phi\|_{L^{2}}^{2}\right] \leqslant \frac{1}{2}\|\varphi\|_{H_{0}^{1}}^{2}+b(\varphi, \phi)_{L^{2}}+\frac{1}{2}\|\phi\|_{L^{2}}^{2} \leqslant \frac{3}{4}\left[\|\varphi\|_{H_{0}^{1}}^{2}+\|\phi\|_{L^{2}}^{2}\right] .
$$

Following [19], it follows from (3), for each $\delta>0$ there is a constant $C_{\delta}>0$ such that

$$
\begin{aligned}
& \int_{\Omega} f(u) u d x \leqslant \delta\|u\|_{L^{2}(\Omega)}^{2}+C_{\delta}, \\
& \int_{\Omega} G(u) d x \leqslant \delta\|u\|_{L^{2}(\Omega)}^{2}+C_{\delta}
\end{aligned}
$$

for each $u \in L^{2}(\Omega)$ such that $f(u) u \in L^{1}(\Omega)$ and $G(u) \in L^{1}(\Omega)$.

Also proceeding as in the proof of Proposition 4.1 in [1], there is a constant $c_{0}>0$ and given $r>0$ there is a constant $c_{r}$ such that

$$
\left.\begin{array}{l}
\left|\int_{\Omega} G(u) d x\right| \leq c_{r}\|u\|_{H_{0}^{1}}^{2} \\
\left|\int_{\Omega} f(u) u d x\right| \leq c_{r}\|u\|_{H_{0}^{1}}^{2}
\end{array}\right\} \quad \text { for all } u \in H_{0}^{1}(\Omega) \text { with }\|u\|_{H_{0}^{1}(\Omega)} \leqslant r \text {. }
$$

From this, if $b$ and $\delta$ are suitably small, there is a constant $K>0$ such that

$$
L_{b}(\phi, \varphi) \geqslant \frac{1}{8}\left[\|\varphi\|_{H_{0}^{1}}^{2}+\|\phi\|_{L^{2}}^{2}\right]-K
$$

and for each $r>0$ a constant $K_{r}>0$ such that

$$
L_{b}(\phi, \varphi) \leqslant K_{r}\left[\|\varphi\|_{H_{0}^{1}}^{2}+\|\phi\|_{L^{2}}^{2}\right]
$$

for all $(\phi, \varphi) \in Y^{0}$ such that $\|(\phi, \varphi)\|_{Y^{0}} \leqslant r$.

Thanks to the regularity of the solutions (see Theorem 2.4), if $\left(u, u_{t}\right)$ denotes the solution of (6) with $\left(u\left(t_{0}\right), u_{t}\left(t_{0}\right)=\left(u_{0}, v_{0}\right)\right.$, we can differentiate the expression $L_{b}\left(u, u_{t}\right)$, 


$$
\begin{aligned}
\frac{d}{d t} L_{b}\left(u, u_{t}\right) & =\left(u_{t t}-\Delta u-f(u), u_{t}\right)_{L^{2}}+b\left\|u_{t}\right\|_{L^{2}}^{2} \\
& +b\left(u, \Delta u+\gamma(t) \Delta u_{t}-\beta_{\varepsilon}(t) u_{t}+f(u)\right)_{L^{2}} \\
& \leqslant-\gamma_{0}\left\|u_{t}\right\|_{H_{0}^{1}}^{2}-\beta_{0 \varepsilon}\left\|u_{t}\right\|_{L^{2}}^{2}-b \gamma(t)\left(u, u_{t}\right)_{H_{0}^{1}}-b\|u\|_{H_{0}^{1}}^{2} \\
& -b \beta_{\varepsilon}(t)\left(u, u_{t}\right)_{L^{2}}+b(u, f(u))_{L^{2}}+b\left\|u_{t}\right\|_{L^{2}}^{2} \\
& \leqslant-\frac{\gamma_{0}}{2}\left\|u_{t}\right\|_{H_{0}^{1}}^{2}-\frac{\lambda_{1} \gamma_{0}}{2}\left\|u_{t}\right\|_{L^{2}}^{2}-\beta_{0 \varepsilon}\left\|u_{t}\right\|_{L^{2}}^{2}-b\|u\|_{H_{0}^{1}}^{2} \\
& +b \gamma_{1}\left(\frac{1}{2 a_{1}}\|u\|_{H_{0}^{1}}^{2}+\frac{a_{1}}{2}\left\|u_{t}\right\|_{H_{0}^{1}}^{2}\right)+b \beta_{1 \varepsilon}\left(\frac{1}{2 a_{2}}\|u\|_{L^{2}}^{2}+\frac{a_{2}}{2}\left\|u_{t}\right\|_{L^{2}}^{2}\right) \\
& +b\left\|u_{t}\right\|_{L^{2}}^{2}+b\left(\delta\|u\|_{H_{0}^{1}}^{2}+C_{\delta}\right) .
\end{aligned}
$$

Hence, for suitable choice of $a_{1}, a_{2}, b$ and $\delta$, there is a $C_{0}>0$ such that

$$
\frac{d}{d t} L_{b}\left(u, u_{t}\right) \leqslant-C_{0}\left(\|u\|_{H_{0}^{1}}^{2}+\left\|u_{t}\right\|_{L^{2}}^{2}\right)+b C_{\delta} .
$$

From (14) with $b=0$, given $r>0$

$$
B_{r}:=\sup \left\{\|u\|_{H_{0}^{1}(\Omega)}^{2}+\left\|u_{t}\right\|_{L^{2}(\Omega)}^{2}: t \geqslant t_{0}, u \in H_{0}^{1}(\Omega) \text { with }\|u\|_{H_{0}^{1}(\Omega)},\left\|u_{t}\right\|_{L^{2}(\Omega)} \leqslant r\right\}<\infty .
$$

Now, if $\left\|\left(u(t), u_{t}(t)\right)\right\|_{Y^{0}}^{2}>\frac{b C_{\delta}}{C_{0}}+\frac{1}{C_{0}}=r_{0}^{2}$ for all $t \geqslant t_{0}$, then there exists a time $T_{r}>0$ such that $L_{b}\left(u(t), u_{t}(t)\right) \leqslant 0$ for each $t \geqslant t_{0}+T_{r}$. In other case, there exists $t_{u}$ such that $\left\|\left(u\left(t_{u}\right), u_{t}\left(t_{u}\right)\right)\right\|_{Y^{0}}^{2} \leqslant \frac{b C_{\delta}}{C_{0}}+\frac{1}{C_{0}}=r_{0}^{2}$ (and let $t_{u}$ be the smallest time with this property), and then $\left\|\left(u(t), u_{t}(t)\right)\right\|_{Y^{0}} \leqslant R_{1}$ for all $t \geqslant t_{0}+t_{u}$, where $R_{1}$ is the radius of a ball containing the set $B_{r_{0}}$ (defined in (16)). If $t_{u} \leqslant T_{r}$, then $\left\|\left(u(t), u_{t}(t)\right)\right\|_{Y^{0}} \leqslant R_{1}$ for all $t \geqslant t_{0}+T_{r}$. If $t_{u}>T_{r}$, then $L_{b}\left(u(t), u_{t}(t)\right) \leqslant 0$ for $t \in\left[t_{0}+T_{r}, t_{0}+t_{u}\right]$ and $\left\|\left(u(t), u_{t}(t)\right)\right\|_{Y^{0}} \leqslant R_{1}$ for all $t \geqslant t_{0}+t_{u}$.

This implies that the ball of radius $R_{0}$ pullback strongly absorbs bounded subsets of $Y^{0}$ where $R_{0}^{2}=\max \left\{R_{1}^{2}, 8 K\right\}$.

Let us now consider the process $\{S(t, s) ; t \geqslant s\}$ generated by our problem in the phase space $Y^{0}$. Recall that $S(t, s)$ is given as follows.

For each initial value $w_{0}:=\left(u_{0}, v_{0}\right) \in Y^{0}$ and each initial time $s \in \mathbb{R}$, system (6) possesses a unique solution which can be written as

$$
S(t, s) w_{0}=T(t, s) w_{0}+U(t, s) w_{0}=\left(\begin{array}{c}
u\left(t, s, w_{0}\right) \\
u_{t}\left(t, s, w_{0}\right)
\end{array}\right)
$$

where $T(t, s)$ is the evolution process associated to the linear part of (6) (i.e. for $f=0$ ), and

$$
U(t, s) w_{0}=\int_{s}^{t} T(t, \tau) F\left(S(\tau, s) w_{0}\right) d \tau
$$

Proceeding as in (14) and using the functional $\tilde{L}_{b}\left(u, u_{t}\right)=\frac{1}{2}\|u\|_{H_{0}^{1}}^{2}+\frac{1}{2}\left\|u_{t}\right\|_{L^{2}}+b\left(u, u_{t}\right)_{L^{2}}$ being now $\left(u, u_{t}\right)$ a solution of the linear part of (6), we can prove that this linear part decays exponentially to zero. The compactness of $U$ follows from the following facts. 
If we assume that $\rho<\frac{n+2}{n-2}$, then we can choose $s \in\left(\frac{1}{2}, 1\right)$ such that $\rho \leqslant \frac{n+2 s}{n-2}$, and therefore we have the following chain of inclusions:

$$
X^{1 / 2} \hookrightarrow L^{2 n /(n-2)} \stackrel{f}{\longrightarrow} L^{2 n /(n+2 s)} \hookrightarrow X^{-s / 2} \subset \subset X^{-1 / 2},
$$

being the last inclusion compact. Thanks to the assumptions on the function $f$, it is clear that $\mathbf{f}$ is compact; this fact implies that $F$ is also compact and, consequently, the operator $U(t, s)$ is compact as well.

Therefore we can apply Theorem 3.2 and Theorem 3.1 to conclude that there exists the pullback attractor $\{\mathscr{A}(t): t \in \mathbb{R}\}$ in $Y^{0}$. Moreover we have that

$$
\bigcup_{t \in \mathbb{R}} \mathscr{A}(t) \quad \text { is bounded in } Y^{0}
$$

and the bound does not depend on $\varepsilon$.

\section{Regularity of the ATtractor}

Thanks to the analysis carried out in our previous section, we have that the pullback attractor is inside a fixed bounded subset of $Y^{0}$. Our aim in this section is to prove more regularity, which will be necessary to obtain a gradient structure for the attractor. First, we will prove that the attractor is a bounded set of $H_{0}^{1}(\Omega) \times H_{0}^{1}(\Omega)$.

Thanks to (19), we have that the attractor can be written as the set of all global bounded solutions

(20) $\{\mathscr{A}(t): t \in \mathbb{R}\}=\left\{\xi: \mathbb{R} \rightarrow Y^{0}\right.$, such that $\xi$ is a global and bounded solution for (1) $\}$.

Hence, if $\xi(\cdot)=\left[\begin{array}{c}u(\cdot) \\ u_{t}(\cdot)\end{array}\right]: \mathbb{R} \rightarrow Y^{0}$ is such that $\xi(t) \in \mathscr{A}(t)$ for all $t \in \mathbb{R}$, then

$$
\xi(t)=T(t, s) \xi(s)+\int_{s}^{t} T(t, \theta) F(\xi(\theta)) d \theta
$$

and we have that it can be written as

$$
\xi(t)=\int_{-\infty}^{t} T(t, \theta) F(\xi(\theta)) d \theta .
$$

Due to Theorem 2.4 $\xi(t) \in C\left(\mathbb{R}, H_{0}^{1}(\Omega) \times H_{0}^{1}(\Omega)\right)$ and $\xi^{\prime}(t) \in C\left(\mathbb{R}, H_{0}^{1}(\Omega) \times L^{2}(\Omega)\right)$. Therefore $\mathscr{A}(t)=S(t, s) \mathscr{A}(s) \subset H_{0}^{1}(\Omega) \times H_{0}^{1}(\Omega)$ for all $t \in \mathbb{R}$.

Now we will proved a higher regularity following the ideas in [5]. If $\xi=\left[\begin{array}{c}u \\ u_{t}\end{array}\right]: \mathbb{R} \rightarrow Y^{0}$ is a global solution of (66) in the pullback attractor and $w_{0}=\xi(s)$, consider

$$
U(t, s):=\left[\begin{array}{c}
w(t) \\
w_{t}(t)
\end{array}\right]=\int_{s}^{t} L(t, \theta) F\left(S(\theta, s) w_{0}\right) d \theta
$$

and note that,

$$
\left\{\begin{array}{l}
w_{t t}-\gamma(t) \Delta w_{t}-\Delta w+\beta_{\varepsilon}(t) w_{t}=f\left(u\left(t, s ; w_{0}\right)\right), \\
w(s)=w_{t}(s)=0 .
\end{array}\right.
$$


Proceeding as before, we can prove that

$$
\{U(t, s): t \geqslant s\} \quad \text { is bounded in } H_{0}^{1} \times L^{2} .
$$

Using the fact that $\mathbf{f}$ takes bounded subsets of $X^{\frac{1}{2}}$ to bounded subsets of $X^{-\frac{1}{2}+\epsilon_{1}}$ (with $\epsilon_{1}$ as in (11)) we can state the problem (6) in $X^{\frac{1}{2}+\epsilon} \times X^{\frac{1}{2}+\epsilon}$ with $\epsilon<\epsilon_{1}$ (note that $U(0)=\left[\begin{array}{l}0 \\ 0\end{array}\right] \epsilon$ $\left.X^{\frac{1}{2}+\epsilon} \times X^{\frac{1}{2}+\epsilon}\right)$. We now use (22) to obtain the uniform bounds for $U(t, s)$ in $X^{\frac{1}{2}+\epsilon} \times X^{\frac{1}{2}+\epsilon}$.

$$
\begin{aligned}
& \|U(t, s)\|_{X^{\frac{1}{2}+\epsilon} \times X^{\frac{1}{2}+\epsilon}} \leqslant \int_{s}^{t}\|L(t, \theta)\|_{\mathcal{L}\left(X^{\frac{1}{2}+\epsilon_{1}} \times X^{\left.-\frac{1}{2}+\epsilon_{1}, X^{\frac{1}{2}+\epsilon} \times X^{\frac{1}{2}+\epsilon}\right)}\right.}\left\|F\left(S(\theta, s) w_{0}\right)\right\|_{X^{\frac{1}{2}+\epsilon_{1} \times X^{-\frac{1}{2}+\epsilon_{1}}}} d \theta \\
& \leqslant K \int_{s}^{t}(t-\theta)^{-1+\epsilon-\epsilon_{1}} e^{-\alpha(t-\theta)} d \theta \text {. }
\end{aligned}
$$

Therefore, noting that $\xi(t)=\lim _{s \rightarrow-\infty} U(t, s)$, we have that

$$
\sup _{\xi \in \mathfrak{A}} \sup _{t \in \mathbb{R}}\left\{\|\xi(t)\|_{X^{\frac{1}{2}+\epsilon} \times X^{\frac{1}{2}+\epsilon}}\right\}<\infty
$$

where $\mathfrak{A}$ is the set of global bounded solutions for (6). We can now repeat this proceedure (since $\mathbf{f}$ takes bounded subsets of $X^{\frac{1}{2}+\epsilon}$ into bounded subsets of $X^{-\frac{1}{2}+\rho \epsilon}$ ) to obtain a bound for the pullback attractor in $X^{\frac{1}{2}+\rho \epsilon} \times X^{\frac{1}{2}+\rho \epsilon}$. In a finite number of steps we arrive at

$$
\sup _{\xi \in \mathfrak{A}} \sup _{t \in \mathbb{R}}\left\{\|\xi(t)\|_{X^{1} \times X^{1}}\right\}<\infty .
$$

Since $\mathbf{f}$ takes bounded subsets of $X^{1}$ in bounded subsets of $X$, we have a uniform bound for $u_{t t}$ in $L^{2}$ inside the attractor noting that

$$
\left\|u_{t t}\right\|_{L^{2}}=\left\|\Delta u+\gamma(t) \Delta u_{t}-\beta_{\varepsilon}(t) u_{t}+f(u)\right\|_{L^{2}}<\infty .
$$

From this we have that

$$
\sup \left\{\|\xi(t)\|_{X^{1} \times X^{1}}+\left\|\xi^{\prime}(t)\right\|_{X^{1} \times X}: \quad \xi \in \mathfrak{A}, t \in \mathbb{R}\right\}<\infty .
$$

Therefore, noting that $\xi(t)=\lim _{s \rightarrow-\infty} U(t, s)$, we have that

$$
\sup _{\xi \in \mathfrak{A}} \sup _{t \in \mathbb{R}}\left\{\|\xi(t)\|_{\left(H^{2} \cap H_{0}^{1}\right) \times H_{0}^{1}},\left\|\xi_{t}(t)\right\|_{Y^{0}}\right\}<\infty
$$

where $\mathfrak{A}$ is the set of global bounded solutions for (66). Noting that $\mathbf{f}$ takes bounded subsets of $H^{2}$ into bounded subsets of $L^{2}$ and using the equation (1) we obtain that

$$
\bigcup_{t \in \mathbb{R}} \mathscr{A}(t) \quad \text { is bounded in } H^{2}(\Omega) \cap H_{0}^{1}(\Omega) \times H^{2}(\Omega) \cap H_{0}^{1}(\Omega) \text {. }
$$




\section{Structure of the Pullback attractor in the limit Problem When $\varepsilon=0$}

Let $\left\{\mathscr{A}_{0}(t): t \in \mathbb{R}\right\}$ be the pullback attractor for (6) when the parameter $\varepsilon=0$. Our aim is to prove the continuity of the attractors showing the upper and lower-semicontinuity. In the second case we will need some particular structure for the limit problem

$$
\left\{\begin{array}{l}
u_{t t}-\gamma(t) \Delta u_{t}-\Delta u=f(u), \text { in } \Omega, \\
u=0, \text { in } \partial \Omega .
\end{array}\right.
$$

We are going to proceed as in [5], therefore we need to assume that there are only finitely many solutions $\left\{u_{1}^{*}, \cdots, u_{p}^{*}\right\}$ of

$$
\left\{\begin{array}{l}
\Delta u+f(u)=0, x \in \Omega \\
u=0, x \in \partial \Omega
\end{array}\right.
$$

Denote by $\mathcal{E}=\left\{e_{1}^{*}, \cdots, e_{p}^{*}\right\}$ where $e_{i}^{*}=\left(\begin{array}{c}u_{i}^{*} \\ 0\end{array}\right)$. Under this assumption, we prove in this section that we can write the limit attractor as

$$
\mathscr{A}_{0}(t)=\bigcup_{i=1}^{p} W^{u}\left(e_{i}^{*}\right)(t), \text { for all } t \in \mathbb{R},
$$

where

$$
\begin{aligned}
& W^{u}\left(e_{i}^{*}\right)=\left\{(\tau, \zeta) \in \mathbb{R} \times Y^{0}: \text { there is a backwards solution } z(t, \tau, \zeta) \text { of (6) }\right) \\
&\text { satisfying } \left.z(t, \tau, \zeta)=\zeta \text { and such that } \lim _{t \rightarrow-\infty}\left\|z(t)-e_{i}^{*}\right\|_{Y^{0}}=0\right\},
\end{aligned}
$$

and $W^{u}\left(e_{i}^{*}\right)(t)=\left\{\zeta:(t, \zeta) \in W^{u}\left(e_{i}^{*}\right)\right\}$.

Due to the fact that $\gamma(\cdot)$ is bounded and Lipschitz, given a sequence $\left\{t_{n}\right\} \subset \mathbb{R}$ we have that $\left\{\gamma\left(t_{n}+t\right)=\gamma_{n}(t)\right\}$ has a convergent subsequence $\gamma_{n}(t) \rightarrow \lambda(t)$ uniformly for $t$ in compact subsets of $\mathbb{R}$, which is also bounded $\left(\gamma_{0} \leqslant \lambda(t) \leqslant \gamma_{1}\right)$ and Lipschitz. Let us define the following problems,

$$
\left\{\begin{array}{l}
u_{t t}-\gamma_{n}(t) \Delta u_{t}-\Delta u=f(u), \text { in } \Omega \\
u=0 \text { in } \partial \Omega \\
u(s)=u_{0} \in H_{0}^{1}(\Omega), u_{t}(s)=v_{0} \in L^{2}(\Omega) .
\end{array}\right.
$$

and

$$
\left\{\begin{array}{l}
u_{t t}-\lambda(t) \Delta u_{t}-\Delta u=f(u), \text { in } \Omega \\
u=0 \text { in } \partial \Omega \\
u(s)=u_{0} \in H_{0}^{1}(\Omega), u_{t}(s)=v_{0} \in L^{2}(\Omega) .
\end{array}\right.
$$

with solutions $\left(u, u_{t}\right)$ and $\left(v, v_{t}\right)$ respectively. Our aim is to compare solutions of the above problems with $\left[\begin{array}{l}u_{0} \\ v_{0}\end{array}\right] \in \mathcal{A}_{n}(s)$ where $\left\{\mathcal{A}_{n}(t): t \in \mathbb{R}\right\}$ is the pullback attractor for (34).

We note that, proceeding exactly as in the previous section we obtain that

$$
\bigcup\left\{\mathscr{A}_{n}(t) \cup \mathscr{A}_{0}(t): n \in \mathbb{N}, t \in \mathbb{R}\right\}
$$


is bounded in $H_{0}^{1} \times H_{0}^{1}$. For $\left[\begin{array}{l}u_{0} \\ v_{0}\end{array}\right] \in \mathcal{A}_{n}(s)$, let $\xi_{n}(t)$ and $\xi_{\infty}(t)$ be the solutions of (34) and (35), respectively.

Defining $z=\xi_{n}-\xi_{\infty}$, we have

$$
\left\{\begin{array}{l}
z_{t t}=\Delta z+\lambda(t) \Delta z_{t}+\left(\gamma_{n}(t)-\lambda(t)\right) \Delta\left(\xi_{n}\right)_{t}+f\left(\xi_{n}\right)-f\left(\xi_{\infty}\right) \text { in } \Omega \\
z=0 \text { in } \partial \Omega .
\end{array}\right.
$$

To prove the convergence of these solutions we consider the following,

$$
\begin{aligned}
\frac{d}{d t}\left[\frac{1}{2}\|z\|_{H_{0}^{1}}^{2}\right. & \left.+\frac{1}{2}\left\|z_{t}\right\|_{L^{2}}^{2}+b\left(z, z_{t}\right)_{L^{2}}\right]=\left(-\Delta z+z_{t t}, z_{t}\right)_{L^{2}}+b\left\|z_{t}\right\|_{L^{2}}^{2}+b\left(z_{t t}, z\right)_{L^{2}} \\
& \leqslant-\gamma_{0}\left\|z_{t}\right\|_{H_{0}^{1}}^{2}-b\|z\|_{H_{0}^{1}}^{2}+\left|\gamma_{n}(t)-\lambda(t)\right|\left|\left(\left(\xi_{n}\right)_{t}, z_{t}\right)_{H_{0}^{1}}\right|+b \lambda(t)\left|\left(z_{t}, z\right)_{H_{0}^{1}}\right| \\
& +b\left|\gamma_{n}(t)-\lambda(t)\right|\left|\left(\left(\xi_{n}\right)_{t}, z\right)_{H_{0}^{1}}\right|+\left|\left(f\left(\xi_{n}\right)-f\left(\xi_{\infty}\right), z_{t}\right)_{L^{2}}\right| \\
& +b\left|\left(f\left(\xi_{n}\right)-f\left(\xi_{\infty}\right), z\right)_{L^{2}}\right| \\
& \leqslant-\frac{\gamma_{0}}{2}\left\|z_{t}\right\|_{H_{0}^{1}}^{2}-\frac{b}{2}\|z\|_{H_{0}^{1}}^{2}+K\left|\gamma_{n}(t)-\lambda(t)\right|,
\end{aligned}
$$

where we have used the bounds in (36) and estimates similar to those in (14) and (16). Therefore,

$$
\|z\|_{H_{0}^{1}}^{2}+\left\|z_{t}\right\|_{L^{2}}^{2} \leqslant K \int_{s}^{t}\left|\gamma_{n}(\theta)-\lambda(\theta)\right| d \tau \leqslant \max _{\theta \in[t, s]}\left|\gamma_{n}(\theta)-\lambda(\theta)\right| K(t-s) \stackrel{n \rightarrow 0}{\longrightarrow} 0,
$$

and we can conclude that, for $t$ in compact subsets of $\mathbb{R}$,

$$
\left\|\xi_{n}(t)-\xi_{\infty}(t)\right\|_{Y^{0}} \stackrel{n \rightarrow \infty}{\longrightarrow} 0 .
$$

We consider the Lyapunov function $\mathcal{L}(\phi, \varphi)=\frac{1}{2}\left(\|\phi\|_{H_{0}^{1}}^{2}+\|\varphi\|_{L^{2}}^{2}\right)-\int_{\Omega} \int_{0}^{\phi(x)} f(s) d s d x$. Then $\mathbb{R} \ni t \mapsto \mathcal{L}\left(\xi(t), \xi_{t}(t)\right) \in \mathbb{R}$ is non-increasing and the only global solutions $\xi$ where $\mathcal{L}$ is constant are the equilibria in $\mathcal{E}$. Since $\{\xi(t): t \in \mathbb{R}\}$ lies in a compact set, there are real numbers $\ell_{i}$ and $\ell_{j}$ such that

$$
\ell_{i} \stackrel{t \rightarrow-\infty}{\longleftarrow} \mathcal{L}(\xi(t+r)) \stackrel{t \rightarrow \infty}{\longrightarrow} \ell_{j}
$$

for all $r \in \mathbb{R}$.

If $t_{n} \stackrel{n \rightarrow \infty}{\longrightarrow} \infty$, taking subsequences if necessary, $\gamma\left(t_{n}+r\right) \stackrel{n \rightarrow \infty}{\longrightarrow} \lambda(r)$ uniformly in compact subsets of $\mathbb{R}, \xi\left(t_{n}+r\right) \stackrel{n \rightarrow \infty}{\longrightarrow} \zeta(r)$ in $Y^{0}$, uniformly for $r$ in compact subsets of $\mathbb{R}$, and $\left(\zeta(t), \zeta_{t}(t)\right)$ is a global solution of the problem

$$
\left\{\begin{array}{l}
u_{t t}-\lambda(t) \Delta u_{t}-\Delta u=f(u), \text { in } \Omega, \\
u=0 \text { in } \partial \Omega,
\end{array}\right.
$$

with the property that $\mathcal{L}\left(\zeta(t), \zeta_{t}(t)\right)=\ell_{j}$, for all $t \in \mathbb{R}$. Hence $\left(\begin{array}{c}\zeta(t) \\ \zeta_{t}(t)\end{array}\right)=e_{j}^{*}$. Taking $\tilde{t}_{n} \stackrel{n \rightarrow \infty}{\longrightarrow}$ $-\infty$, we obtain an analogous result.

Suppose that there are sequences $\left\{t_{n}\right\}_{n \in \mathbb{N}}$ and $\left\{\bar{t}_{n}\right\}_{n \in \mathbb{N}}$ with $t_{n+1}>\bar{t}_{n}>t_{n}, n \in \mathbb{N}$, such that $\xi\left(t_{n}\right) \stackrel{n \rightarrow \infty}{\longrightarrow} e_{k}^{*}$ and $\xi\left(\bar{t}_{n}\right) \stackrel{n \rightarrow \infty}{\longrightarrow} \bar{e}_{k}^{*}$. Now, given $\epsilon>0$, there exists $n_{\epsilon} \in \mathbb{N}$ such that 
$\mathcal{L}(\xi(t)) \in\left(\ell_{j}-\epsilon, \ell_{j}+\epsilon\right)$ for all $t \in\left[t_{n}, \bar{t}_{n}\right]$. If $\tau_{n} \in\left(t_{n}, \bar{t}_{n}\right), \tau_{n} \stackrel{n \rightarrow \infty}{\longrightarrow} \infty$ and (taking subsequences if necessary), $\gamma\left(\tau_{n}+r\right) \stackrel{n \rightarrow \infty}{\longrightarrow} \bar{\lambda}(r)$. We have that $\xi\left(\tau_{n}+r\right) \stackrel{n \rightarrow \infty}{\longrightarrow} \bar{\zeta}(t)$, which is a solution of

$$
\left\{\begin{array}{l}
u_{t t}-\bar{\lambda}(t) \Delta u_{t}-\Delta u=f(u), \text { in } \Omega, \\
u=0 \text { in } \partial \Omega,
\end{array}\right.
$$

with $\mathcal{L}\left(\bar{\zeta}(t), \bar{\zeta}_{t}(t)\right)=\ell_{j}$ for all $t \in \mathbb{R}$, and, consequently, $\bar{\zeta}(t) \equiv e_{m}^{*}$ with $\mathcal{L}\left(e_{m}^{*}\right)=\ell_{j}$. That leads to a contradiction with the fact that there are only finitely many equilibria. Therefore we can write the pullback attractor as in (32).

\section{Continuity of the attractors}

In this section we prove the continuity of the pullback attractors for (1) when $\beta_{\varepsilon}(t) \stackrel{\varepsilon \rightarrow 0}{\longrightarrow} 0$. From now on, we will denote $\left\{S_{\varepsilon}(t, s): t \geqslant s\right\}$ the process associated to (6) and $\left\{\mathscr{A}_{\varepsilon}(t): t \in\right.$ $\mathbb{R}\}$ the pullback attractor of $S_{\varepsilon}(t, s)$ for each $\varepsilon$.

6.1. Upper-semicontinuity. Let $U_{0} \in H_{0}^{1} \times L^{2}, v=S_{0}(t+s, s) U_{0}, u=S_{\varepsilon}(t+s, s) U_{0}$ and $w=u-v$, where $\left\{S_{0}(t, s): t \geqslant s\right\}$ is the evolution process associated to the limit problem (30). We have that

$$
\left\{\begin{array}{l}
w_{t t}=\Delta w+\gamma(t) \Delta w_{t}-\beta_{\varepsilon}(t) u_{t}+f(u)-f(v), \\
w(s)=w_{t}(s)=0
\end{array}\right.
$$

Let us consider the functional $H\left(w, w_{t}\right)=\frac{1}{2}\left(\|w\|_{H_{0}^{1}}^{2}+\left\|w_{t}\right\|_{L^{2}}^{2}\right)$. Then, there is a constant $K>0$ such that

$$
\begin{aligned}
\frac{d}{d t} H\left(w, w_{t}\right) & =\left(w_{t t}-\Delta w, w_{t}\right)_{L^{2}} \\
& \leqslant-\gamma_{0}\left\|w_{t}\right\|_{H_{0}^{1}}^{2}+\beta_{\varepsilon}(t)\left(u_{t}, w_{t}\right)_{L^{2}}+\left(f(u)-f(v), w_{t}\right)_{L^{2}} \\
& \leqslant K H\left(w, w_{t}\right)+K \beta_{1 \varepsilon},
\end{aligned}
$$

where we used that $f: H_{0}^{1} \rightarrow L^{2 n / n+2}$ is Lipschitz, Hölder's inequality and (36). By the Gronwall's Lemma,

$$
\|w\|_{H_{0}^{1}}^{2}+\left\|w_{t}\right\|_{L^{2}}^{2} \leqslant C \beta_{1 \varepsilon} \int_{s}^{t} e^{K(t-\tau)} d \tau \stackrel{\varepsilon \rightarrow 0}{\longrightarrow} 0,
$$

in compact subsets of $\mathbb{R}$, uniformly for $U_{0}$ in bounded subsets of $H_{0}^{1} \times L^{2}$.

Let $\tau \in \mathbb{R}$ be such that $\operatorname{dist}\left(S_{0}(t, \tau) \mathcal{B}, \mathscr{A}_{0}(t)\right)<\frac{\delta}{2}$ where $\bigcup_{s \in \mathbb{R}} \mathscr{A}_{\varepsilon}(s) \subset \mathcal{B}$ for all $\delta>0$. Therefore, using (42), there exists $\epsilon_{0}>0$ such that $\sup _{a_{\epsilon} \in \mathscr{A}_{\epsilon}}\left\|S_{\varepsilon}(t, \tau) a_{\varepsilon}(\tau)-S_{0}(t, \tau) a_{\varepsilon}(\tau)\right\|<\frac{\delta}{2}$ for all $\epsilon \leqslant \epsilon_{0}$. Then,

$$
\begin{aligned}
\operatorname{dist}\left(\mathscr{A}_{\varepsilon}(t), \mathscr{A}_{0}(t)\right) & \leqslant \sup _{a_{\epsilon} \in \mathscr{A}_{\epsilon}(\tau)} d\left(S_{\varepsilon}(t, \tau) a_{\varepsilon}, S_{0}(t, \tau) a_{\varepsilon}\right)+\operatorname{dist}\left(S_{0}(t, \tau) \mathscr{A}_{\varepsilon}(\tau), \mathscr{A}_{0}(t)\right) \\
& <\frac{\delta}{2}+\frac{\delta}{2}=\delta
\end{aligned}
$$

and the upper-semicontinuity is proved. 
6.2. Lower-semicontinuity. First of all, we need to suppose that all equilibrium points in $\mathcal{E}$ are hyperbolic for the limit problem in the following sense.

Definition 6.1. We say that the linear evolution process $\left\{L_{i}(t, \tau): t \geqslant \tau \in \mathbb{R}\right\}$ has exponential dichotomy with exponent $\omega$ and constant $M$ if there is a family of bounded linear projections $\left\{Q_{i}(t): t \in \mathbb{R}\right\}$ in $X$ such that

(1) $Q_{i}(t) L_{i}(t, s)=L_{i}(t, s) Q_{i}(s)$, for all $t \geqslant s$.

(2) The restriction $L_{i}(t, s)_{\left.\right|_{R\left(Q_{i}(s)\right)}}, t \geqslant s$ is an isomorphism from $R\left(Q_{i}(s)\right)$ into $R\left(Q_{i}(t)\right)$; we denote its inverse by $\left[L_{i}(t, s)\right]^{-1}=L_{i}(s, t)$ with $L_{i}(s, t): R\left(Q_{i}(t)\right) \rightarrow R\left(Q_{i}(s)\right)$ (notice that $R\left(Q_{i}(t)\right)$ denotes the range of the operator $\left.Q_{i}(t)\right)$.

(3) There are constants $\omega>0$ and $M \geqslant 1$ such that

$$
\begin{aligned}
& \left\|L_{i}(t, s)\left(I-Q_{i}(s)\right)\right\|_{L(X)} \leqslant M e^{-\omega(t-s)} \quad t \geqslant s \\
& \left\|L_{i}(t, s) Q_{i}(s)\right\|_{L(X)} \leqslant M e^{\omega(t-s)}, \quad t \leqslant s .
\end{aligned}
$$

When $\left\{L_{i}(t, \tau): t \geqslant \tau \in \mathbb{R}\right\}$ possesses an exponential dichotomy, we say that $e_{i}^{*}$ is a hyperbolic equilibrium point.

The key of the proof of the lower-semicontinuity is based on the proof of the local continuity of the sets $W_{0}^{u}\left(e_{i}^{*}\right)$ and $W_{\varepsilon}^{u}\left(e_{i}^{*}\right)$ defined as in (33), written them first as a graph and showing later the continuity. Our aim is to parallel the analysis carried out in Section 2 from [9], using the following theorem.

Theorem 6.2. Let $X$ be a Banach space and considerer the family $\left\{S_{\varepsilon}(t, s): t \geqslant s\right\}$, $\varepsilon \in[0,1]$, of nonlinear processes in $X$. Assume that for any $x$ in a compact subset of $X$, $\left\|S_{\varepsilon}(t, s) x-S_{0}(t, s) x\right\| \stackrel{\varepsilon \rightarrow 0}{\longrightarrow} 0$ for $[t, s] \subset \mathbb{R}$ and suppose that for each $\varepsilon \in[0,1]$ there exits a pullback attractor $\left\{\mathscr{A}_{\varepsilon}(t): t \in \mathbb{R}\right\}$, such that $\overline{\bigcup_{t \in \mathbb{R}} \bigcup_{\varepsilon \in\left[0, \varepsilon_{0}\right]} \mathscr{A}_{\varepsilon}(t)}$ is compact and we can write $\left\{\mathscr{A}_{0}(t): t \in \mathbb{R}\right\}$ as in (32). Further, assume that for each $e_{j}^{*} \in \mathcal{E}$ :

(1) given $\delta>0$, there exists $\varepsilon_{j, \delta}$, such that for all $0<\varepsilon<\varepsilon_{j, \delta}$ there is a global hyperbolic solution $\xi_{j, \varepsilon}$ of (6) that satisfies

$$
\sup _{t \in \mathbb{R}}\left\|\xi_{j, \varepsilon}(t)-e_{j}^{*}\right\|_{X}<\delta,
$$

(2) the local unstable manifold of $\xi_{j, \varepsilon}$ behaves continuously as $\varepsilon \rightarrow 0$; that is,

$$
\operatorname{dist}_{H}\left(W_{0, l o c}^{u}\left(e_{j}^{*}\right), W_{\varepsilon, l o c}^{u}\left(\xi_{j, \varepsilon}\right)\right) \rightarrow 0,
$$

where $\operatorname{dist}_{H}(A, B)=\max \{\operatorname{dist}(A, B), \operatorname{dist}(B, A)\}$ is the symmetric Hausdorff distance and $W_{\text {loc }}^{u}(\cdot)=W^{u}(\cdot) \cap B_{X}(\cdot, \rho)$, with $\rho>0$.

Then the family $\left\{\mathscr{A}_{\varepsilon}(t): t \in \mathbb{R}, 0 \leqslant \varepsilon \leqslant \varepsilon_{0}\right\}$ is lower-semicontinuous at $\varepsilon=0$, i.e.

$$
\operatorname{dist}\left(\mathscr{A}_{0}(\mathrm{t}), \mathscr{A}_{\varepsilon}(\mathrm{t})\right) \stackrel{\varepsilon \rightarrow 0}{\longrightarrow} 0 .
$$

The compactness of the union of all pullback attractors is obtained in (29) and, in Section 5. we have proved that the pullback attractor of the limit problem is gradient-like. Therefore, we need to prove the two conditions in Theorem 6.2. These are just consequences of the stability of the hyperbolic equilibria under perturbation (see [8]). 
We then need to prove that all the equilibrium points in $\mathcal{E}$ are hyperbolic for all $S_{\varepsilon}(t, s)$. If $u(t)$ is a solution of (30) and defining $z(t)=u(t)-e_{j}^{*}$ for any $e_{j}^{*} \in \mathcal{E}$, then $z(\cdot)$ satisfies

$$
\left\{\begin{array}{l}
z_{t t}-\Delta z-\gamma(t) \Delta z_{t}-f^{\prime}\left(e_{j}^{*}\right) z=h(z) \\
z(s)=z_{0} ; z_{t}(s)=z_{1}
\end{array}\right.
$$

where $h(z)=f\left(z+e_{j}^{*}\right)-f\left(e_{j}^{*}\right)-f^{\prime}\left(e_{j}^{*}\right) z$. Then, we can construct the following system

$$
\left(\begin{array}{c}
z \\
x
\end{array}\right)_{t}+\overline{\mathbf{A}}_{0}(t)\left(\begin{array}{c}
z \\
x
\end{array}\right)=H\left(\begin{array}{c}
z \\
x
\end{array}\right)
$$

where

$$
\overline{\mathbf{A}}_{0}(t)=\left(\begin{array}{cc}
0 & -I \\
-\Delta-f^{\prime}\left(e_{j}^{*}\right) & -\gamma(t) \Delta
\end{array}\right) \text { and } H\left(\begin{array}{c}
z \\
x
\end{array}\right)=\left(\begin{array}{c}
0 \\
h(z)
\end{array}\right) .
$$

In the same way, taking $v(t)$ solution of (1) and defining $z(t)=v(t)-e_{j}^{*}$ we have

$$
\left(\begin{array}{l}
z \\
x
\end{array}\right)_{t}+\left[\overline{\mathbf{A}}_{0}(t)+B_{\varepsilon}(t)\right]\left(\begin{array}{l}
z \\
x
\end{array}\right)=H\left(\begin{array}{l}
z \\
x
\end{array}\right)
$$

where

$$
B_{\varepsilon}(t)=\left(\begin{array}{cc}
0 & 0 \\
0 & \beta_{\varepsilon}(t)
\end{array}\right)
$$

with $H(0) \equiv 0$ and the Jacobian matrix $J_{H}(0) \equiv 0 \in \mathcal{L}\left(Y^{0}\right)$.

Let $\left\{Z_{0}(t, s): t \geqslant s\right\}$ and $\left\{Z_{\varepsilon}(t, s): t \geqslant s\right\}$ denote the processes associated to (46) and (47) respectively. Note that

$$
\sup _{t \in \mathbb{R}}\left\|B_{\varepsilon}(t)\right\|_{\mathcal{L}\left(Y^{0}\right)}=\sup _{t \in \mathbb{R}}\left|\beta_{\varepsilon}(t)\right| \leqslant \beta_{1 \varepsilon} \stackrel{\varepsilon \rightarrow 0}{\longrightarrow} 0,
$$

then, by Theorem 7.6.11 in [18], it follows that for $\varepsilon_{0}>0$ sufficiently small, $\left\{Z_{\varepsilon}(t, s): t \geqslant s\right\}$ has an exponential dichotomy and, consequently, every point in $\mathcal{E}$ is also hyperbolic for $\left\{S_{\varepsilon}(t, s): t \geqslant s\right\}$, for all $\varepsilon<\varepsilon_{0}$.

Therefore we can apply Theorem [6.2, obtaining the lower-semicontinuity of the pullback attractors.

Remark 6.3. Observe that, in particular, we have proved upper and lower semicontinuity of pullback attractors with respect to the autonomous strongly damped wave equation, i.e., the one with $\gamma(t)=\gamma>0$.

Acknowledgements. We would like to thank the referee for his/her interesting remarks and suggestions.

\section{REFERENCES}

[1] J. Arrieta, A. N. Carvalho and J. K. Hale, A damped hyperbolic equation with critical exponent, Communications in Partial Differential Equations, 17 (5-6) (1992) 841-866.

[2] A.B. Babin, M.I. Vishik, Attractors of evolution equations. North Holland (1992).

[3] S.M. Bruschi, A.N. Carvalho, J.W. Cholewa, T. Dlotko, Uniform Exponential Dychotomy and Continuity of Attractors for Singular Perturbed Damped Equations, J. of Dynamics and Differential Eq. 18 3 (2006) 767-814. 
[4] T. Caraballo, A.N. Carvalho, J.A. Langa, F. Rivero, Existence of pullback attractors for pullback asymptotically compact processes, Nonlinear Analysis, 72 (2010) 1967-1976.

[5] T. Caraballo, A.N. Carvalho, J.A. Langa, F. Rivero, Some gradient-like non-autonomous evolution processes, Int. Journal of Bifurcation and Chaos, Vol. 20, No. 9 (2010). DOI: 10.1142/S0218127410027337.

[6] A.N. Carvalho, J.W. Cholewa, Local well-posedness for strongly damped wave equation with critical nonlinearities, Bull. Austral. Math. Soc, 66 (2002) 443-463.

[7] A.N. Carvalho, J.W. Cholewa, T. Dlotko, Strongly damped wave problems: Bootstrapping and regularity of solutions, J. Differential Equations, 244 (2008) 2310-2333.

[8] A.N. Carvalho, J.A. Langa, Non-autonomous perturbation of autonomous semilinear differential equations: Continuity of local stable and unstable manifolds, J. Differential Equations, 233 (2007) 622-653.

[9] A.N. Carvalho, J.A. Langa, J.C. Robinson, Lower semicontinuity of attractors for non-autonomous dynamical systems, Ergodic Theory Dynam. Systems 29, 6 (2009) 1765-1780.

[10] A.N. Carvalho, J.A. Langa, J.C. Robinson, On the continuity of pullback attractors for evolution processes, Nonlinear Analysis, 71 (2009) 1812-1824.

[11] A.N. Carvalho, M.J. Nascimento, Singularly non-autonomous semilinear parabolic problems with critical exponents, Discrete Contin. Dyn. Syst. Ser. S 23 (2009) 449-471.

[12] D.N. Cheban, Global Attractors of Non-Autonomous Dissipative Dynamical Systems. World Scientific Publishing Co. Pte. Ltd (2004).

[13] S. Chen, R. Triggiani, Proof of extensions of two conjetures on structural damping for elastic systems, Pacific J. of Mathematics 1351 (1989) 15-55.

[14] S. Chen, R. Triggiani, Characterization of domains of fractional powers of certain operators arising in elastic systems, and applications, J. Differential Equations 88, 2 (1990) 279-293.

[15] V. V. Chepyzhov, M.I. Vishik, Attractors for Equations of Mathematical Physics. Colloquium Publications, Vol. 49. America Mathematical Society (2002).

[16] P.L. Christiansen, P.S. Lomdhal, O.H. Soerensen, Soliton excitations in Josephson tunnel junctions, Phys. Rev. 25 (1982) 5737-5748.

[17] G. Duvaut, J-L. Lions, Inequalities in Mechanics and Physics. Springer (1976).

[18] D. Henry, Geometry Theory of Semilinear Parabolic Equations, Springer-Verlag (1981).

[19] J.K. Hale, Asymptotic Behavior of Dissipative System, American Mathematical Society (1989).

[20] F. Hirosawa, J. Wirth, Generalised energy conservation law for wave equations with variable propagation speed, J. Math. Anal. Appl., 358 (2009) 56-74.

[21] V. Kalantarov, S. Zelik, Finite-dimensional attractors for the quasi-linear strongly-damped wave equation, 247 (2009) 1120-1155.

[22] P. E. Kloeden, Pullback attractors of nonautonomous semidynamical system. Stochastics and Dynamics 3, 1 (2003) 101-112.

[23] K. Nishihsra, J. Zhai, Asymptotic behavior of solutions for time dependent damped wave equations, J. Math. Anal. Appl, 360 (2009) 412-421.

[24] V. Pata, M. Squassina, On the Strongly Damped Wave equation, Commum. Math. Phys, 253 (2005) $511-533$.

[25] V. Pata, S. Zelik, Smooth attractors for strongly damped wave equation, Nonlinearity, 19 (2006) 14951506.

[26] G. R. Sell, Nonautonomous differential equations and dynamical systems. Trans. Amer. Math. Soc. 127 (1967) 241-283.

[27] P. E. Sobolevskiŭ, Equations of parabolic type in a Banach space, in: Amer. Math. Soc. Transl. Series 2, Vol. 49: Ten Papers on Functional Analysis and Measure Theory, American Mathematical Society, Providence, R.I., (1966), 162.

(T. Caraballo) Departamento de Ecuaciones Diferenciales y Análisis Numérico, Universidad de Sevilla, Apdo. de Correos 1160, 41080-Sevilla Spain 
(A. N. Carvalho) Instituto de Ciências Matemáticas e de ComputaÇao, Universidade de SÃo Paulo-Campus de São Carlos, Caixa Postal 668, 13560-970 São Carlos SP, Brazil

(J. A. Langa) Departamento de Ecuaciones Diferenciales y Análisis Numérico, Universidad de Sevilla, Apdo. de Correos 1160, 41080-Sevilla Spain

(F. Rivero) Departamento de Ecuaciones Diferenciales y Análisis Numérico, Universidad de Sevilla, Apdo. De Correos 1160, 41080-Sevilla Spain 